\title{
Global strong solutions in Sobolev or Lebesgue spaces to the incompressible Navier-Stokes equations in $\mathbb{R}^{3}$
}

by

\section{F. PLANCHON}

Centre de Mathématiques, U.R.A. 169 du C.N.R.S., Ecole Polytechnique, F-91128 Palaiseau Cedex

ABSTRACT. - We construct global strong solutions of the Navier-Stokes equations with sufficiently oscillating initial data. We will show that the condition is for the norm in some Besov space to be small enough.

RÉSUMÉ. - Nous construisons des solutions fortes globales des équations de Navier-Stokes, pour des données initiales suffisamment oscillantes. Cette condition se traduit en terme de norme petite dans un certain espace de Besov.

\section{INTRODUCTION}

We are interested in the following system, for $x \in \mathbb{R}^{3}$ and $t>0$,

$$
\left\{\begin{array}{l}
\frac{\partial u}{\partial t}+(u \cdot \nabla) u=\nu \Delta u-\nabla p \\
\nabla \cdot u=0,
\end{array}\right.
$$

with initial data $u(x, 0)=u_{0}(x)$.For the sake of simplicity, we suppose that $\nu=1$; a simple rescaling allows us to obtain any other value. Local 
existence and uniqueness in the Sobolev space $H^{s}\left(\mathbb{R}^{3}\right)$ and the Lebesgue space $L^{p}\left(\mathbb{R}^{3}\right)$ are known, if $s>1 / 2$ and $p>3$ (see [4]). We have global solutions for small initial data in $L^{3}\left(\mathbb{R}^{3}\right)$ (see [9] or [4]) and $H^{\frac{1}{2}}\left(\mathbb{R}^{3}\right)$ (see [4] and [5]), or in $L^{2}\left(\mathbb{R}^{3}\right) \cap L^{p}\left(\mathbb{R}^{3}\right)$, with $p>3$ (see [1]). We shall extend the results of [4], for $s>1 / 2$ and $p>3$. By adapting the auxiliary spaces used in [4], we shall prove the existence and uniqueness of global solutions in $H^{s}\left(\mathbb{R}^{3}\right)$ provided the initial data are small in a sense which will be made precise later, and in $L^{p}\left(\mathbb{R}^{3}\right)$ up to additional conditions on $u_{0}$. Let us define the homogeneous Besov spaces $\dot{B}_{p, q}^{\alpha}$ :

DEFINITION 1. - Let us choose $\phi \in \mathcal{S}\left(\mathbb{R}^{n}\right)$ a radial function so that Supp $\hat{\phi} \subset\{|\xi|<1+\varepsilon\}$, and $\hat{\phi}(\xi)=1$ for $|\xi|<1$. Define $\phi_{j}(x)=$ $2^{n j} \phi\left(2^{j} x\right), S_{j}$ the convolution operator with $\phi_{j}$, and $\Delta_{j}=S_{j+1}-S_{j}$. Let $f \in \mathcal{S}^{\prime}\left(\mathbb{R}^{n}\right), \alpha \in \mathbb{R}, 1<p, q \leq+\infty, f \in \dot{B}_{p, q}^{\alpha}$ if and only if

$$
\left[\sum_{-\infty}^{+\infty}\left(2^{j \alpha}\left\|\Delta_{j} f\right\|_{L^{p}}\right)^{q}\right]^{\frac{1}{q}}<+\infty .
$$

The reader should consult [12], [2], or [16] where the properties of Besov spaces are exposed in detail. Let us see how homogeneous Besov spaces arise. If we want to construct a global solution, it is useful to control a norm remaining invariant by the rescaling $f(x) \rightarrow \lambda f(\lambda x)$. If this can be achieved in a Besov space with $\alpha<0$ and therefore bigger than the usual space where we want to obtain a solution, we will have weaker assumptions on $u_{0}$.

Let us give the results in the case of Sobolev spaces. $B C$ denotes the class of bounded continuous functions.

THEOREM 1. - There exists an universal constant $\beta>0$ such that, if $s>\frac{1}{2}, u_{0} \in H^{s}\left(\mathbb{R}^{3}\right), \nabla \cdot u_{0}=0$ and

$$
\|u\|_{\dot{B}_{4, \infty}^{-1 / 4}}<\beta
$$

then there exists a unique solution $u$ of (1) such that

$$
u \in B C\left([0, \infty), H^{s}\left(\mathbb{R}^{3}\right)\right) .
$$

Moreover, the following properties hold for $u$ :

- $\|u(\cdot, t)\|_{L^{2}}$ is decreasing, and for every $t>1$,

$$
\left\|u(\cdot, t)-e^{t \Delta} u_{0}\right\|_{L^{2}} \leq \frac{C\left(\beta, u_{0}\right)}{t^{1 / 4}}
$$


- For every $t>1$,

$$
\left\|(-\Delta)^{s / 2} u(\cdot, t)\right\|_{L^{2}} \leq \frac{C\left(\beta, u_{0}, s\right)}{t^{s / 2}} .
$$

- For every $t>0$,

$$
\|u(\cdot, t)\|_{\infty} \leq \frac{C\left(\beta, u_{0}\right)}{\sqrt{t}}
$$

- If $s \in(1,3 / 2]$, for every $t<1$,

$$
\left\|u(\cdot, t)-e^{t \Delta} u_{0}\right\|_{\infty} \leq C\left(\beta, u_{0}\right) .
$$

Note that the space $\dot{B}_{4, \infty}^{-1 / 4}$ is invariant under the scaling $f(x) \rightarrow \lambda f(\lambda x)$, and $\dot{H}^{\frac{1}{2}} \subset \dot{B}_{4, \infty}^{-1 / 4}$. It is very interesting that we do not need a small $H^{\frac{1}{2}}$ norm to obtain a global solution (see [4]). On the other hand, if we want to include the case $1 / 2, u$ is unique in the space

$$
\left\{\begin{array}{l}
u \in B C\left([0,+\infty), H^{\frac{1}{2}}\right) \\
t^{1 / 8} u(\cdot, t) \in B C\left([0,+\infty), L^{4}\right) \\
\lim _{t \rightarrow 0} t^{1 / 8}\|u\|_{L^{4}}=0 .
\end{array}\right.
$$

which was used in [4], the starting point of the present work. The weak condition (2) is the only remaining obstacle to the problem of existence of global smooth solutions to the Navier-Stokes equations, and we remark that $\beta$ does not depend on $s$. The decay estimates (4) can be found in [8], in a slightly different context. We recall it here as a natural consequence of the construction of $u$.

In the Lebesgue spaces, the analogue is

THEOREM 2. - Let $p>3 / 2$, there exists $\delta(p)>0$ such that, if $u_{0} \in L^{p} \cap \dot{B}_{2 p, \infty}^{-\left(1-\frac{3}{2 p}\right)}, \nabla \cdot u_{0}=0$ and

$$
\left\|u_{0}\right\|_{\dot{B}_{2 p, \infty}^{-\left(1-\frac{3}{2 p}\right)}}<\delta(p)
$$

then there exists a unique solution $u$ such that

$$
\left\{\begin{array}{l}
u \in B C\left([0,+\infty), L^{p}\right) \\
t^{\frac{1}{2}-\frac{3}{4 p}} u(\cdot, t) \in B C\left([0,+\infty), L^{2 p}\right) \\
\lim _{t \rightarrow 0} t^{\frac{1}{2}-\frac{3}{4 p}}\|u\|_{L^{2 p}}=0 .
\end{array}\right.
$$


The restriction $p>3 / 2$ is due to technical considerations, and we could probably obtain 1 instead of $3 / 2$, by sligthly modifying the Besov space involved.

Proposition 1. - The constant $\delta(p)$ satisfies:

$$
\begin{gathered}
\lim _{p \rightarrow+\infty} \delta(p)=0, \\
\lim _{p \rightarrow 3 / 2} \delta(p)=0 .
\end{gathered}
$$

Proposition 2. - In Theorem 2, we can replace $u_{0} \in L^{p} \cap \dot{B}_{2 p, \infty}^{-\left(1-\frac{3}{2 p}\right)}$ by $u_{0} \in L^{p} \cap L^{3}$, and if $p>3$ by $L^{2} \cap L^{p}$.

If $u_{0} \in H^{s}, s \geq 1 / 2$, then as $\dot{H}^{\frac{1}{2}} \subset \dot{B}_{4, \infty}^{-1 / 4}$, we have a natural candidate for the useful Besov space. On the contrary, if we take $L^{p}$, we may use two different Besov spaces: the first one is $\dot{B}_{2 p, \infty}^{-\frac{3}{2 p}}$, as $L^{p} \subset \dot{B}_{2 p, \infty}^{-\frac{3}{2 p}}$. But this space is not invariant by the rescaling. The "right" space is $\dot{B}_{2 p, \infty}^{-\left(1-\frac{3}{2 p}\right)}$, but unfortunately $L^{p} \not \subset \dot{B}_{2 p, \infty}^{-\left(1-\frac{3}{2 p}\right)}$. This explains the additional condition imposed on $u_{0}$ in Theorem 2 . Both spaces coincide only when $1-\frac{3}{2 p}=\frac{3}{2 p}$, which means $p=3$. The reader should refer to [9] and [4] for details.

Proofs. - We first reformulate the problem in order to obtain an integral equation for $u$. This is standard practice, and was first employed by Kato and Fujita (see [10] [11]), and very often used since (see [7] [6] [15]). All these authors use semi-group theory, but in the present case, we do not need this formalism, for the exact expression of the heat kernel in $\mathbb{R}^{3}$ allows us to obtain directly the estimates we need (see [9]). Let $\mathbb{P}$ be the projection operator from $\left(L^{2}\left(\mathbb{R}^{3}\right)\right)^{3}$ onto the subspace of divergence-free vectors, denoted by $\mathbb{P} L^{2}$, and $R_{j}$ the Riesz transform with symbol $\frac{\xi_{j}}{|\xi|}$. We easily see that

$$
\mathbb{P}\left(\begin{array}{l}
u_{1} \\
u_{2} \\
u_{3}
\end{array}\right)=\left(\begin{array}{l}
u_{1} \\
u_{2} \\
u_{3}
\end{array}\right)-\left(\begin{array}{l}
R_{1} \sigma \\
R_{2} \sigma \\
R_{3} \sigma
\end{array}\right)
$$

where $\sigma=\sum_{j} R_{j} u_{j}$.It is well-known that $\mathbb{P}$ can be extended to a bounded operator from $\left(L^{p}\right)^{3}$ onto $\mathbb{P} L^{p}, 1<p<+\infty$, and from $\left(H^{s}\right)^{3}$ onto $\mathbb{P} H^{s}$, $s \geq 0$. Note that $\mathbb{P}$ commutes with $S(t)=e^{t \Delta}$, whereas on an open set $\Omega$, we need to introduce the Stokes operator $-\mathbb{P} \Delta$ and the associated semi-group. Note that

$$
\operatorname{Ker} \mathbb{P}=\{u \mid \exists \phi \text { such that } u=\nabla \phi\} .
$$


Using $\mathbb{P},(1)$ becomes an evolution equation

$$
\left\{\begin{aligned}
\frac{\partial u}{\partial t} & =\Delta u-\mathbb{P} \nabla \cdot(u \otimes u), \\
\nabla \cdot u & =0 \\
u(x, 0) & =u_{0}(x)
\end{aligned}\right.
$$

We replace $(u \cdot \nabla) u$ by $\nabla \cdot(u \otimes u)$ to avoid problems of definition, and this is possible only because $\nabla \cdot u=0$. It is then standard to study (10) via the corresponding integral equation

$$
u(x, t)=S(t) u_{0}(x)-\int_{0}^{t} \mathbb{P} S(t-s) \nabla \cdot(u \otimes u)(x, s) d s
$$

in a space of divergence free vectors. The integral should be seen as a Bochner integral. In the general case of evolution equations, a solution of (11) might not be a solution of (10). However, in the case of the Navier-Stokes equations without external forces, it is true without any extra assumptions. Actually, the solutions of (11) are $C^{\infty}\left((0,+\infty) \times \mathbb{R}^{3}\right)$ and verify the equations (1) in the classical sense, as we recover easily the pressure up to a constant by

$$
-\Delta p=\sum_{i, j} \frac{\partial u_{i}}{\partial x_{j}} \frac{\partial u_{j}}{\partial x_{i}}
$$

The reader should refer to [7] [10] or [13] for proofs. We remark that since a solution of (1) is necessarily a solution of (11), uniqueness for (11) guarantees uniqueness for (1). We aim to solve (11) by successive approximations, with the following lemma:

LeMma 1. - Let $E$ and $F$ be two Banach functional spaces, endowed with the norms $\|\cdot\|=\|\cdot\|_{E}$ and $|\cdot|=\|\cdot\|_{F}, B$ a continuous bilinear operator from $F \times F \rightarrow E$ and $F \times F \rightarrow F$ :

$$
\begin{gathered}
\|B(u, v)\| \leq \eta|u \| v| \\
|B(u, v)| \leq \gamma|u \| v|
\end{gathered}
$$

and define the sequence $X_{0}=0, X_{n+1}=Y+B\left(X_{n}, X_{n}\right)$, where $Y$ belongs to $E$ and to $F$. If

$$
4 \gamma|Y|<1
$$

Vol. 13, $\mathrm{n}^{\circ} 3-1996$. 
then the sequence converges in both spaces $E$ and $F$, and the limit $X$ sastisfies

$$
X=Y+B(X, X)
$$

and

$$
|X|<2|Y|
$$

The proof is left to the reader. Note that the value of $\eta$ has no influence on the convergence. Now we have to study the following bilinear operator

$$
B(u, v)=\int_{0}^{t} \mathbb{P} S(t-s) \nabla \cdot(u \otimes u) d s .
$$

In order to simplify the notations, we limit ourselves to the following scalar operator

$$
B(f, g)=\int_{0}^{t} \frac{1}{(t-s)^{2}} \theta\left(\frac{\cdot}{(t-s)^{2}}\right) * f g(s) d s
$$

As $\mathbb{P} S(t-s) \nabla \cdot$ is a matrix of convolution operators, the components are all operators like (17), with

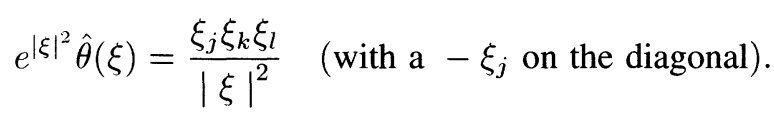

Lemma 2. $-\theta(x) \in C^{\infty}\left(\mathbb{R}^{3}\right)$ and $\theta \in L^{1} \cap L^{\infty}$.

This can be easily seen on the Fourier transform of $\theta$.

In what follows, $C$ denotes a constant which may vary from one line to another.

\section{Proof of Theorem 1}

Proposition 3. - Let $1 / 2<s \leq 3 / 4$, then there exists a solution $u$ of (11) such that

$$
\left\{\begin{aligned}
u & \in B C\left([0,+\infty), \dot{H}^{s}\right)=E \\
\omega(t) u(x, t) & \in B C\left([0,+\infty), L^{4}\right)=F
\end{aligned}\right.
$$

where $\omega(t)=t^{3 / 8-s / 2}$ if $0<t<1$ and $\omega(t)=t^{1 / 8}$ if $t \geq 1$. 
We want to apply Lemma (1) where $\mathrm{E}$ and $\mathrm{F}$ are defined by the norms

$$
\begin{aligned}
& \|u\|=\sup _{t}\|u\|_{\dot{H}^{s}} \\
& |u|=\sup _{t} \omega(t)\|u\|_{L^{4}} .
\end{aligned}
$$

If we use Hölder and Young inequalities for $B(f, g), \Lambda$ being the operator with symbol $|\xi|$,

(20) $\left\|\Lambda^{s} B(f, g)(t)\right\|_{L^{2}} \leq\|\theta\|_{L^{1}}|f \| g| \int_{0}^{t} \frac{1}{(t-\tau)^{1 / 2+s / 2} \omega^{2}(\tau)} d \tau$,

$$
\|B(f, g)(t)\|_{L^{4}} \leq\|\theta\|_{L^{4 / 3}}|f \| g| \int_{0}^{t} \frac{1}{(t-\tau)^{7 / 8} \omega^{2}(\tau)} d \tau .
$$

We shall then verify that, for all $t>0$,

$$
\begin{aligned}
& I_{1}=\int_{0}^{t} \frac{1}{(t-\tau)^{1 / 2+s / 2} \omega^{2}(\tau)} d \tau<+\infty, \\
& I_{2}=\int_{0}^{t} \frac{1}{(t-\tau)^{7 / 8} \omega^{2}(\tau)} d \tau<+\infty .
\end{aligned}
$$

Easy calculations actually show that for $t<1$,

$$
I_{i}<C t^{s / 2-1 / 4}
$$

and for $t>1$

$$
I_{i}<C t^{1 / 4-s / 2}
$$

The continuity at $t=0$ comes from the estimate when $t<1$. In order to include the case $s=1 / 2$, we have to impose $\lim _{t \rightarrow 0} t^{1 / 8}\|u\|_{L^{4}}=0$ (see [4]). Note that the constant $\gamma$ of Lemma 1 is

$$
\gamma=\int_{0}^{1} \frac{\|\theta\|_{L^{4 / 3}}}{(1-\tau)^{7 / 8} \tau^{1 / 4}} d \tau
$$

Therefore, if $S(t) u_{0}$ satisfies condition (13), we obtain $u \in$ $B C\left([0,+\infty), \dot{H}^{s}\right)$.

PROPOSITION 4. - We have

$$
u \in B C\left([0,+\infty), L^{2}\right) .
$$

Vol. 13, n ${ }^{\circ}$ 3-1996. 
Let $G=B C\left([0,+\infty), L^{2}\right) ; B$ is bicontinuous from $G \times F$ to $G$ :

$$
\|B(f, g)(t)\|_{L^{2}} \leq|g| \sup _{[0, t]}\|f\|_{L^{2}}\|\theta\|_{L^{4 / 3}} \int_{0}^{t} \frac{1}{(t-\tau)^{7 / 8} \omega(\tau)} d \tau \text {. }
$$

Let

$$
I_{3}=\int_{0}^{t} \frac{\|\theta\|_{L^{4 / 3}}}{(t-\tau)^{7 / 8} \omega(\tau)} d \tau
$$

for $t<1, I_{3}<C t^{s / 2-1 / 4}$, and for all $t$

$$
I_{3} \leq \int_{0}^{1} \frac{\|\theta\|_{L^{4 / 3}}}{(1-\tau)^{7 / 8} \tau^{7 / 8}} d \tau=\rho .
$$

$G$ being a Banach space, we can use a contraction argument to show that the sequence defined previously converges in $G$. It is sufficient that $2|u| \rho<1$, which is true as $\rho \leq \gamma$ and $u$ verifies (15). Therefore, we proved (24) and hence Proposition 3, and shown that $\|u(\cdot, t)\|_{L^{2}}$ is uniformly bounded.

We now show (6): the following estimation is verified by the heat kernel,

$$
\sup _{[0, t]} \sqrt{t}\left\|S(t) u_{0}\right\|_{\infty} \leq C .
$$

We have

$$
\|B(f, g)(t)\|_{\infty} \leq\|\theta\|_{L^{4 / 3}} \int_{0}^{t} \frac{\|f(s)\|_{L^{4}}\|g(s)\|_{\infty}}{(t-s)^{7 / 8}} d s .
$$

Let us denote $W(f, t)=\sup _{[0, t]} \sqrt{t}\|f(., s)\|_{\infty}$, then

$$
W(B(f, g), t) \leq|f| W(g, t)\|\theta\|_{L^{4 / 3}} \int_{0}^{t} \frac{\sqrt{t}}{(t-s)^{7 / 8} s^{5 / 8}} d s .
$$

Let

$$
I_{4}=\int_{0}^{1} \frac{\|\theta\|_{L^{4 / 3}}}{(1-\mu)^{7 / 8} \mu^{5 / 8}} d \mu
$$

then, as $I_{4} \leq 2 \gamma$, we have $2 W\left(S(t) u_{0}, t\right) I_{4}<1$. Therefore,

$$
\sup _{[0, t]} \sqrt{t}\|u(\cdot, t)\|_{\infty} \leq \frac{C}{1-2 I_{4}\left\|S(t) u_{0}\right\|} .
$$

Now we can prove (4) as follows:

$$
\|B(f, g)(t)\|_{L^{2}} \leq \int_{0}^{t} \frac{C}{(t-s)^{2-\frac{3}{2 q}}}\|g\|_{L^{2}}\|f\|_{L^{\beta}} d s,
$$


where

$$
\frac{1}{2}=\frac{1}{q}+\frac{1}{2}+\frac{1}{\beta}-1 .
$$

If we take $q$ such that $\frac{3}{2 q}=1+\varepsilon, \varepsilon>0$, using interpolation and (28) we get, for $t>1$,

$$
\|f\|_{L^{p}} \leq\|f\|_{L^{2}}{ }^{\frac{2}{p}}\|f\|_{\infty}{ }^{1-\frac{2}{p}}
$$

and

$$
\begin{aligned}
\|B(f, g)(t)\|_{L^{2}} & \leq \int_{0}^{t} \frac{C}{(t-s)^{1-\varepsilon} s^{\frac{1}{6}+\frac{2}{3} \varepsilon}} d s \\
\|B(f, g)(t)\|_{L^{2}} & \leq \frac{C(\varepsilon)}{t^{\frac{1}{6}-\frac{\varepsilon}{3}}} .
\end{aligned}
$$

On the other hand, we know by (26) that $\forall q \geq 2$,

$$
\sup _{[0, t]} t^{\frac{3}{2}\left(\frac{1}{2}-\frac{1}{q}\right)}\left\|S(t) u_{0}\right\|_{q} \leq C .
$$

Therefore, as $u$ sastisfies (14), we will improve (30) in the following way: let

$$
\begin{aligned}
& B_{1}(f, g)=\int_{0}^{1} \frac{1}{(t-s)^{2}} \theta\left(\frac{\cdot}{\sqrt{t-s}}\right) * f g(s) d s \\
& B_{2}(f, g)=\int_{1}^{t} \frac{1}{(t-s)^{2}} \theta\left(\frac{\cdot}{\sqrt{t-s}}\right) * f g(s) d s .
\end{aligned}
$$

The term $B_{1}$ can be handled very easily, so that $\forall \eta>0$,

$$
\left\|B_{1}(f, g)(t)\right\|_{L^{2}} \leq \frac{C}{t^{1-\eta}}
$$

Now, we split $B_{2}(u, u)$ in three parts. By (31) we have

$$
\begin{aligned}
\left\|B_{2}\left(S(t) u_{0}, S(t) u_{0}\right)\right\|_{L^{2}} & \leq \int_{1}^{t} \frac{C}{(t-s)^{2-\frac{3}{2 q}} s^{\frac{3}{2}\left(1-\frac{1}{\alpha}-\frac{1}{\beta}\right)}} d s \\
& \leq \frac{C}{t^{1-\frac{3}{2 q}+\frac{3}{2}\left(1-\left(\frac{3}{2}-\frac{1}{q}\right)\right)}} \\
& \leq \frac{C}{t^{1 / 4}}
\end{aligned}
$$

Vol. 13, $\mathrm{n}^{\circ} 3-1996$. 
as

$$
\frac{1}{2}=\frac{1}{\gamma}+\frac{1}{\alpha}+\frac{1}{\beta}-1 .
$$

We remark that the exponent $1 / 4$ cannot be improved, as it does not depend on $\gamma, \alpha$ and $\beta$.

Lemma 3. - Suppose that for $0<\mu$

$$
\|B(u, u)(t)\|_{L^{2}} \leq \frac{C}{t^{\mu}}
$$

then

$$
\left\|B_{2}\left(S(t) u_{0}, B(u, u)\right)\right\|_{L^{2}} \leq \frac{C}{t^{1 / 4+\mu}},
$$

and there exists $\nu>0$ such that

$$
\left\|B_{2}(B(u, u), B(u, u))\right\|_{L^{2}} \leq \frac{C}{t^{\mu+\nu}} .
$$

By (31)

$$
\begin{aligned}
\left\|B_{2}\left(S(t) u_{0}, B(u, u)\right)\right\|_{L^{2}} & \leq \int_{1}^{t} \frac{C}{(t-s)^{2-\frac{3}{2 q}} s^{\frac{3}{2}\left(\frac{1}{2}-\frac{1}{3}\right)} s^{\mu}} d s \\
& \leq \frac{C}{t^{-\varepsilon+\frac{3}{2}\left(\frac{1}{6}+\frac{2}{3} \varepsilon\right)+\mu}} \\
& \leq \frac{C}{t^{1 / 4+\mu}}
\end{aligned}
$$

and, by (28) and (29)

$$
\begin{aligned}
\left\|B_{2}(B(u, u), B(u, u))\right\|_{L^{2}} & \leq \int_{1}^{t} \frac{C}{(t-s)^{2-\frac{3}{2 q}} s^{\mu} s^{\frac{1}{2}\left(1-\frac{2}{\beta}\right)+\frac{2}{\beta} \mu}} d s \\
& \leq \frac{C}{t^{-\frac{1}{3} \varepsilon+\frac{1}{6}+\left(\frac{5}{3}-\frac{4}{3} \varepsilon\right) \mu}}
\end{aligned}
$$

We can start with $\mu=1 / 6-\varepsilon$, and obtain any exponent $\eta>1 / 4$. Thus,

$$
\left\|B_{2}(u, u)\right\|_{L^{2}} \leq \frac{C}{t^{1 / 4}}
$$

We constructed $u$ for $s \leq 3 / 4$. Now we will see that if $s>3 / 4, u$ as above is actually in $H^{s}$. We limit ourselves to the bilinear form (17), as the term $S(t) u_{0}$ satisfies at least the same estimates. 
LeMmA 4. - Let $f, g \in H^{s}\left(\mathbb{R}^{3}\right), 3 / 4 \leq s<3 / 2$,

$$
\left\|\Lambda^{2 s-3 / 2}(f g)\right\|_{L^{2}} \leq C\left\|\Lambda^{s} f\right\|_{L^{2}}\left\|\Lambda^{s} g\right\|_{L^{2}}
$$

For a proof see the Appendix. Suppose now that $s>3 / 4$, and $u$ is the solution of Proposition 3 for $s=3 / 4$. Then, if $\eta \leq 1 / 4$, we obtain for $t<1$

$$
\left\|\Lambda^{\frac{3}{4}+\eta} B(f, g)(t)\right\|_{L^{2}} \leq \int_{0}^{t} \frac{C}{(t-\tau)^{\frac{1}{2}+\frac{3 / 4+\eta}{2}}} d \tau
$$

using Lemma 4 and the boundedness of $f$ in $H^{3 / 4}$, so that

$$
\left\|\Lambda^{\frac{3}{4}+\eta} B(f, g)(t)\right\|_{L^{2}} \leq C t^{\frac{1}{8}-\frac{\eta}{2}}
$$

which gives the continuity at zero. For $t>1$, we have by (29)

$$
\|B(f, g)\|_{L^{4}} \leq \frac{C}{t^{1 / 4}}
$$

which allows us to improve (22), for $s \leq 3 / 4$

$$
\begin{aligned}
\left\|\Lambda^{s} B(f, g)(t)\right\|_{L^{2}} & \leq \int_{0}^{1} \frac{\|\theta\|_{L^{1}}}{(t-\tau)^{1 / 2+s / 2} \omega^{2}(\tau)} d \tau|f \| g| \\
& +\int_{1}^{t} \frac{C\|\theta\|_{L^{1}}}{(t-\tau)^{1 / 2+s / 2} \tau^{1 / 2}} d \tau \\
& \leq \frac{C}{t^{s / 2}} .
\end{aligned}
$$

Then

$$
\left\|\Lambda^{\frac{3}{4}} f(t)\right\|_{L^{2}} \leq \frac{C}{t^{\frac{3}{8}}}
$$

and,

$$
\left\|\Lambda^{\frac{3}{4}+\eta} B_{1}(f, g)\right\|_{L^{2}} \leq \frac{C}{(t-1)^{\frac{1}{2}+\frac{3 / 4+\eta}{2}}}
$$

and

$$
\left\|\Lambda^{\frac{3}{4}+\eta} B_{2}(f, g)\right\|_{L^{2}} \leq \int_{1}^{t} \frac{C}{(t-\tau)^{\frac{1}{2}+\frac{3 / 4+\eta}{2}} \tau^{\frac{1}{2}}} d \tau
$$

then, for all $t$

$$
\left\|\Lambda^{\frac{3}{4}+\eta} B(f, g)(t)\right\|_{L^{2}} \leq \frac{C}{1+t^{\frac{3}{8}+\frac{\eta}{2}}}
$$

Vol. $13, \mathrm{n}^{\circ} 3-1996$. 
We have thus obtained $u \in H^{\frac{3}{4}+\eta}$. By applying the same argument we can reach the value $s>3 / 2$, as

$$
\Lambda^{s+\eta} B(f, g)(t)=\int_{0}^{t} \frac{1}{(t-\tau)^{2}} \Lambda^{\eta+3 / 2-s} \theta * \Lambda^{2 s-3 / 2}(f g)(\tau) d \tau,
$$

with $\eta+3 / 2-s<1$. Before dealing with the case $s>3 / 2$, let us briefly show (7) . By Sobolev's injection theorem (see [14]), if $s<3 / 2$ then

$$
\|f\|_{L^{p}} \leq C\left\|\Lambda^{s} f\right\|_{L^{2}}
$$

with $1 / p=1 / 2-s / 3$. If $s=1+\alpha, \alpha<1 / 2$, we obtain, for $t<1$

$$
\|B(f, g)(t)\|_{\infty} \leq \int_{0}^{t} \frac{C}{(t-\tau)^{1-\alpha}} d \tau
$$

For small $t, B(f, g)$ is bounded and tends to zero as $t$ goes to zero. Now we treat the case where $s>3 / 2$, using the following estimate

Lemma 5. - Let $f, g \in H^{s}\left(\mathbb{R}^{3}\right), s>3 / 2$,

$$
\left\|\Lambda^{s}(f g)\right\|_{L^{2}} \leq C(s)\left(\left\|\Lambda^{s} f\right\|_{L^{2}}\|g\|_{\infty}+\left\|\Lambda^{s} g\right\|_{L^{2}}\|f\|_{\infty}\right)
$$

For a proof, see the appendix. We will then show

LEMma 6. - Let $s>3 / 2$, for all $t>0$

$$
\left\|\Lambda^{s} u(\cdot, t)\right\|_{L^{2}} \leq \frac{C(s)}{1+t^{\frac{s}{2}}} .
$$

This can be achieved by successive iterations, starting from the previous estimate for $s=2$, and applying Lemma 5. Let us see how it works at each step. Let $\eta<1$, we first treat the case $t<1$.

$$
\left\|\Lambda^{s+\eta} B(f, g)(t)\right\|_{L^{2}} \leq \int_{0}^{t} \frac{1}{(t-\tau)^{2}}\left\|\Lambda^{\eta} \theta\left(\frac{\cdot}{\sqrt{t-\tau}}\right) * \Lambda^{s}(f g)\right\|_{L^{2}} d \tau
$$

and as $f$ and $g$ are bounded in $L^{\infty}$ and in $H^{s}$,

$$
\left\|\Lambda^{s+\eta} B(f, g)(t)\right\|_{L^{2}} \leq C(s) t^{\frac{1}{2}-\frac{\eta}{2}} .
$$

For $t>1$,

$$
\begin{aligned}
& \left\|\Lambda^{s+\eta} B_{1}(f, g)\right\|_{L^{2}} \\
& \quad \leq \int_{0}^{1} \frac{1}{(t-\tau)^{\frac{1}{2}+\frac{s+\eta-1 / 2}{2}}}\left\|\Lambda^{s+\eta-\frac{1}{2}} \theta\right\|_{L^{1}}\|\Lambda f\|_{L^{2}}\|\Lambda g\|_{L^{2}} d \tau
\end{aligned}
$$


by using Lemma 4 . Then

$$
\left\|\Lambda^{s+\eta} B_{1}(f, g)\right\|_{L^{2}} \leq \frac{C(s)}{(t-1)^{\frac{s+\eta+1 / 2}{2}}} .
$$

For $t>1$,

$$
\left\|\Lambda^{s+\eta} B_{2}(f, g)\right\|_{L^{2}} \leq \int_{1}^{t} \frac{1}{(t-\tau)^{2}}\left\|\Lambda^{\eta} \theta\left(\frac{\cdot}{\sqrt{t-\tau}}\right) * \Lambda^{s}(f g)\right\|_{L^{2}} d \tau
$$

and using Lemma 5 we deduce the estimate for $s+\eta$ from the estimate for $s$ :

$$
\left\|\Lambda^{s+\eta} B_{2}(f, g)\right\|_{L^{2}} \leq \frac{C(s)}{t^{\frac{s+\eta}{2}}} .
$$

This achieves the proof of the existence of $u \in B C\left([0,+\infty), H^{s}\right)$. Now, we observe that, as we have local existence and uniqueness for $s>1 / 2$ (see [4]), our solution is unique by applying this theorem on intervals covering $[0, \infty)$. In the case $s=1 / 2$, it is necessary to establish uniqueness directly, (see [4] or [11]). The reader should refer to [11] or [7], in order to see why a solution of (11) is actually a solution in the classical sense. We can nevertheless make a few remarks. By the same process we use to gain the regularity $s-3 / 4$, we can establish, independently of $s$, estimates in $H^{r}, r>s$ : for all $t>0$, there exists $\pi(r)>0$

$$
\left\|\Lambda^{r} u(\cdot, t)\right\|_{L^{2}} \leq \frac{C(r)}{t^{\pi(r)}}
$$

and $\Lambda^{r} u$ is holderian on every interval $\left[t_{0}, t_{1}\right]$, provided $t_{1}>t_{0}>0$.This provides the regularity in the space variables. As for regularity in time, it suffices to use the relation, which can be established without knowing (10)

$$
u(t)=S(t-\varepsilon) u(\varepsilon)+\int_{\varepsilon}^{t} \mathbb{P} S(t-\tau) \nabla \cdot(u \otimes u) d \tau,
$$

and the following lemma, (see [11] or [7] for a proof).

Lemma 7. - Let $u(t)=\int_{0}^{t} e^{-(t-s) \Delta} f(s) d s, t \in[0, T], f \in C^{\eta}([0, T], B)$, $\eta<1, B$ a Banach space. Then $u \in C^{1+\nu}((0, T], B), A u \in C^{\nu}((0, T], B)$, and

$$
\partial_{t} u=-\Delta u+f
$$

for all $\nu<\eta$.

We then obtain the $C^{\infty}$ regularity of $u$, for $t>0$, with a bootstrap argument. Let us see how condition (13) can be expressed on $u_{0}$ in terms Vol. 13, n ${ }^{\circ} 3-1996$. 
of Besov spaces. We set $\left|S(t) u_{0}\right|<\beta$, where $\beta$ has been chosen so that our scheme converges in $F$. Remember that

$$
\left|S(t) u_{0}\right|=\sup _{t} \omega(t)\left\|S(t) u_{0}\right\|_{L^{4}} .
$$

Therefore, as $3 / 8-\inf (s, 3 / 4) / 2<1 / 8$,

$$
\sup _{t} t^{3 / 8-\inf (s, 3 / 4) / 2}\left\|S(t) u_{0}\right\|_{L^{4}}<\beta
$$

and

$$
\sup _{t} t^{1 / 8}\left\|S(t) u_{0}\right\|_{L^{4}}<\beta
$$

LEMMA 8. - Let $u_{0} \in \mathcal{S}\left(\mathbb{R}^{3}\right), \alpha>0$, and $\gamma>1$; $\sup _{t} t^{\alpha / 2}\left\|S(t) u_{0}\right\|_{L^{\gamma}}$ is a norm on $\dot{B}_{\beta, \infty}^{-\alpha}$ which is equivalent to the classical dyadic norm.

We refer to [4] or [12] for a proof. In our case, except for $s=3 / 4$, the condition on $u_{0}$ is equivalent to

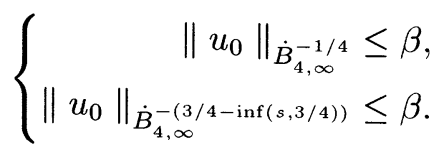

Thus, as $\dot{H}^{\frac{1}{2}} \subset \dot{B}_{4, \infty}^{-1 / 4}$ and $\dot{H}^{\inf (s, 3 / 4)} \subset \dot{B}_{4, \infty}^{-(3 / 4-\inf (s, 3 / 4))}, u_{0}$ belongs to both Besov spaces. If $u$ is a solution with initial condition $u_{0}, \lambda u\left(\lambda x, \lambda^{2} t\right)$ is a solution with $\lambda u_{0}(\lambda x)$ as initial data. The condition (44) is independent of $\lambda$ for the norm is invariant by scaling. And (43) can be forced by a suitable choice of $\lambda$. For $s=3 / 4$, we know that $H^{\frac{3}{4}} \subset L^{4}$, and we conclude in the same way. This ends the proof.

Proof of Theorem 2. - We introduce as before two Banach spaces $E=B C\left([0,+\infty), L^{p}\right)$ with the natural norm

$$
\|f\|=\sup _{t}\|f(\cdot, t)\|_{L^{p}}
$$

and $F=\left\{f \mid t^{\frac{1}{2}-\frac{3}{4 p}} f \in B C\left([0,+\infty), L^{2 p}\right)\right\}$ with the norm

$$
|f|=\sup _{t} t^{\frac{1}{2}-\frac{3}{4 p}}\|f(\cdot, t)\|_{L^{2 p}} \text {. }
$$

Then, we see that

(46) $\|B(f, g)(t)\|_{L^{2 p}} \leq|f||g|\|\theta\|_{L^{q}} \int_{0}^{t} \frac{1}{(t-s)^{2-\frac{3}{2 q}} s^{1-\frac{3}{2 p}}} d s$,

$$
\text { where } \frac{1}{p}=\frac{1}{q}+\frac{1}{p}+\frac{1}{2 p}-1 \text {. }
$$


which gives the continuity of $B$ from $F \times F \rightarrow F$ and $F \times E \rightarrow E$, with constants $\gamma(p)$ and $\eta(p)$.

$$
\begin{aligned}
& \gamma(p)=\|\theta\|_{L^{q}} t^{\frac{1}{2}-\frac{3}{4 p}} \int_{0}^{t} \frac{1}{(t-s)^{2-\frac{3}{2 q}} s^{1-\frac{3}{2 p}}} d s \\
& \eta(p)=\|\theta\|_{L^{q}} \int_{0}^{t} \frac{1}{(t-s)^{2-\frac{3}{2 q}} s^{\frac{1}{2}-\frac{3}{4 p}}} d s
\end{aligned}
$$

and a simple rescaling shows both quantities are bounded. Then if we use the same sequence as before, Lemma 1 gives us the convergence in $F$, and we obtain the convergence in $E$ by acontraction argument, as $\eta(p) \leq \gamma(p)$, we obtain $2|u| \eta(p)<1$. The continuity at $t=0$ comes from a slight modification of (45), as we can replace $|f|$ by $\sup _{[0, t]} \tau^{\frac{1}{2}-\frac{3}{4 p}}\|f(\cdot, \tau)\|_{L^{2 p}}$, which tends to zero with $t$. Actually, the value of $t^{\frac{1}{2}-\frac{3}{4 p}}\|f(\cdot, t)\|_{L^{2 p}}$ could only be zero: the first term $u_{1}=S(t) u_{0}$ tends to zero, for if we consider a sequence of $C_{0}^{\infty}$ functions $\left(v_{j}\right)_{j}$ which approximate $u_{0}$,

$t^{\frac{1}{2}-\frac{3}{4 p}}\left\|S(t) u_{0}\right\|_{L^{2 p}}=t^{\frac{1}{2}-\frac{3}{4 p}}\|S(t)\|\left\|u_{0}-v_{j}\right\|_{L^{p}}+\|S(t)\| t^{\frac{1}{2}-\frac{3}{4 p}}\left\|v_{j}\right\|_{L^{2} p}$.

By Lemma 8 the condition on $u_{0}$ becomes,

$$
\left\|u_{0}\right\|_{\dot{B}_{2 p, \infty}^{-\left(1-\frac{3}{2 p}\right)}} \leq \delta(p),
$$

where $\delta(p) \approx 1 / \gamma(p)$. This proves Proposition 1. Proposition 2 results from the inclusion of $L^{3}$ in $\dot{B}_{2 p, \infty}^{-\left(1-\frac{3}{2 p}\right)}$. Note that for $p=2$, we impose the condition

$$
t^{\frac{1}{4}}\left\|S(t) u_{0}\right\|_{L^{4}}<\delta
$$

which is equivalent to the condition (2). For a general $u_{0} \in L^{2}$, we only know

$$
t^{\frac{3}{8}}\left\|S(t) u_{0}\right\|_{L^{4}}<+\infty
$$

In other words, we do not know enough on low frequencies, and a sufficient condition is (2), of which $u_{0} \in L^{3}$ or $u_{0} \in H^{\frac{1}{2}}$ with small norms are particular cases. We obtained existence and uniqueness in a ball of $F$ with Lemma 1 and uniqueness in the whole space can be obtained directly as in [11] or [4]. As in the Sobolev case, it is possible to obtain estimates on $L^{q}$ norms of $u(\cdot, t), q>p$, in order to show the $C^{\infty}$ regularity for $t>0$. 


\section{APPENDIX}

We recall that if $\phi \in \mathcal{S}\left(\mathbb{R}^{n}\right)$ is a radial function so that Supp $\hat{\phi} \subset\{|\xi|<$ $1+\varepsilon\}$, and $\hat{\phi}(\xi)=1$ for $|\xi|<1$, we define $\phi_{j}(x)=2^{n j} \phi\left(2^{j} x\right), S_{j}$ the convolution operator with $\phi_{j}$, and $\Delta_{j}=S_{j+1}-S_{j}$. Then

$$
I=\sum_{-\infty}^{+\infty} \Delta_{j}
$$

and $f(x) \in \dot{H^{s}}\left(\mathbb{R}^{n}\right)$ if and only if, $\forall j$,

$$
\left\|\Delta_{j}(f)\right\|_{L^{2}} \leq 2^{-j s}\|f\|_{\dot{H}^{s}} \varepsilon_{j}
$$

where $\sum \varepsilon_{j}^{2} \leq 1$. We will show the two following inequalities, which are homogeneous variants of well-known inequalities:

for $s<\frac{n}{2}$,

$$
\left\|\Lambda^{2 s-\frac{n}{2}}(f g)\right\|_{L^{2}} \leq C\left\|\Lambda^{s} f\right\|_{L^{2}}\left\|\Lambda^{s} g\right\|_{L^{2}}
$$

for $s>\frac{n}{2}$,

$$
\left\|\Lambda^{s}(f g)\right\|_{L^{2}} \leq C(s)\left(\left\|\Lambda^{s} f\right\|_{L^{2}}\|g\|_{\infty}+\left\|\Lambda^{s} g\right\|_{L^{2}}\|f\|_{\infty}\right) .
$$

Let us start with the first case: we will use a paraproduct decomposition (see [3]): for $f, g \in \mathcal{S}$,

$$
\begin{aligned}
f(x) g(x) & =\sum_{j} \Delta_{j}(f) \sum_{l} \Delta(g) \\
& =\sum_{|j-l| \leq 1} \Delta_{j}(f) \Delta_{l}(g)+\sum_{|j-l| \geq 1} \Delta_{j}(f) \Delta_{l}(g) .
\end{aligned}
$$

The second sum is, by reordering the terms, a finite sum of terms like $S_{2}=\sum_{j} S_{j-1} \Delta_{j}(g)$. We will treat only $S_{2}$, as the other ones are of the same kind. The Fourier transform of $S_{2}$ is supported in an annulus $\left[2^{j-1}(1-2 \varepsilon), 2^{j+1}(1+2 \varepsilon)\right]$. Using Bernstein's lemma,

$$
\begin{aligned}
\left\|\Delta_{j}(f)\right\|_{\infty} & \leq C 2^{j \frac{n}{2}}\left\|\Delta_{j} f\right\|_{L^{2}} \\
& \leq C 2^{j \frac{n}{2}-s}\|f\|_{\dot{H}^{s}} \varepsilon_{j} .
\end{aligned}
$$

Then,

$$
\left\|S_{j}(f)\right\|_{\infty} \leq C \sum_{-\infty}^{j} 2^{q\left(\frac{n}{2}-s\right)} \varepsilon_{q}\|f\|_{\dot{H}^{s}} .
$$


If $j<0$,

$$
\sum_{-\infty}^{j} 2^{q\left(\frac{n}{2}-s\right)} \varepsilon_{q}=2^{j\left(\frac{n}{2}-s\right)} \tilde{\varepsilon_{j}}
$$

and

$$
\tilde{\varepsilon}_{j}=\sum_{-\infty}^{0} 2^{q\left(\frac{n}{2}-s\right)} \varepsilon_{j+q}
$$

is a convolution product between $l^{1}$ and $l^{2}$, therefore in $l^{2}$. For $j \geq 0$,

$$
\sum_{-\infty}^{j} 2^{q\left(\frac{n}{2}-s\right)} \varepsilon_{q} \leq C\left(1+\ldots+2^{j\left(\frac{n}{2}\right)} \varepsilon_{j}\right) .
$$

if

$$
2^{j\left(\frac{n}{2}-s\right)} \breve{\varepsilon}_{j}=1+\ldots+2^{j\left(\frac{n}{2}-s\right)} \varepsilon_{j},
$$

$\left(\breve{\varepsilon_{j}}\right)$ is in $l^{2}$ for the same reason as $\tilde{\varepsilon_{j}}$. This gives

$$
\left\|S_{j}(f)\right\|_{\infty} \leq C 2^{j\left(\frac{n}{2}-s\right)}\|f\|_{\dot{H}^{s}} \eta_{j} .
$$

where $\left(\eta_{j}\right)_{j} \in l^{2}$. Then, if $\left(\mu_{j}\right)_{j}$ is associated to $g$,

$$
\left\|S_{j-1}(f) \Delta_{j}(g)\right\|_{L^{2}} \leq 2^{j\left(\frac{n}{2}-s\right)}\|f\|_{\dot{H}^{s}}\|g\|_{\dot{H}^{s}} \eta_{j} \mu_{j}
$$

and as $\left(\eta_{j} \mu_{j}\right)_{j} \in l^{1} \subset l^{2}, S_{1} \in \dot{H}^{2 s-\frac{n}{2}}$. The terms of the first sum in (51) are like $S_{1}=\sum_{j} \Delta_{j}(f) \Delta_{j}(g)$, and in this case we only know that the support of the Fourier transform of $\Delta_{j}(f) \Delta_{j}(g)$ is in $\left\{|\xi| \leq C 2^{j}\right\}$, and

$$
\left\|\Delta_{j}(f) \Delta_{j}(g)\right\|_{L^{1}} \leq \varepsilon_{j} \mu_{j} 2^{-2 j s}\|f\|_{\dot{H}^{s}}\|g\|_{\dot{H}^{s}} .
$$

Lemma 9. - If $u \in L^{1}$, supp $\hat{u} \subset B(0, R)$, and $\|\hat{u}(\xi)\|_{\infty} \leq R^{-2 s}$, then

$$
\left\|\Lambda^{2 s-\frac{n}{2}} u\right\|_{L^{2}} \leq \int_{S^{2}} d S .
$$

This comes from

$$
\begin{aligned}
\int_{|\xi| \leq R}\left(|\xi|^{2}\right)^{2 s-\frac{n}{2}}|\hat{u}(\xi)|^{2} d \xi & \leq R^{-4 s} \int_{|\xi| \leq R}|\xi|^{4 s-n} d \xi \\
& \leq R^{-4 s} \int_{S^{2}} \int_{0}^{R} r^{4 s-1} d r d S .
\end{aligned}
$$

then, applying Lemma 9 to $\Delta_{j}(f) \Delta_{j}(g)$,

$$
\left\|S_{j-1}(f) \Delta_{j}(g)\right\|_{H^{2 \dot{s}-\frac{n}{2}}} \leq C \varepsilon_{j} \mu_{j}\|f\|_{\dot{H}^{s}}\|g\|_{\dot{H}^{s}} .
$$

As $\left(\eta_{j} \mu_{j}\right)_{j} \in l^{1}$, this ends the proof. The second inequality can be proved by the same estimates, except that we have a better estimate for $\left\|S_{j}(f)\right\|_{\infty}$ and $\left\|\Delta_{j}(f)\right\|_{\infty}$, both bounded by $\|f\|_{\infty}$. 


\section{REFERENCES}

[1] H. BeIRÃ O DA VEGA, Existence and Asymptotic Behaviour for Strong Solutions of the Navier-Stokes Equations in the Whole Space, Indiana Univ. Math. Journal, Vol. 36(1), 1987, pp. 149-166.

[2] J. BerGH and J. LöFSTROM, Interpolation Spaces, An Introduction, Springer-Verlag, 1976.

[3] J. M. BonY, Calcul symbolique et propagation des singularités dans les équations aux dérivées partielles non linéaires, Ann. Sci. Ecole Norm. Sup., Vol. 14, 1981, pp. 209-246.

[4] M. Cannone, Ondelettes, Paraproduits et Navier-Stokes, PhD thesis, Universite Paris IX, CEREMADE F-75775 PARIS CEDEX, 1994, to be published by Diderot Editeurs (1995).

[5] J.-Y. Chemin, Remarques sur l'existence globale pour le système de Navier-Stokes incompressible, SIAM Journal Math. Anal., Vol. 23, 1992, pp. 20-28.

[6] Y. Giga, Solutions for Semi-Linear Parabolic Equations in $L^{p}$ and Regularity of Weak Solutions of the Navier-Stokes System, Journal of differential equations, Vol. 61, 1986, pp. 186-212.

[7] Y. Giga and T. MiYakawa, Solutions in $L^{r}$ of the Navier-Stokes Initial Value Problem, Arch. Rat. Mech. Anal., Vol. 89, 1985, pp. 267-281.

[8] R. KaJikiYa and T. MiYakawa, On $L^{2}$ Decay of Weak Solutions of the Navier-Stokes Equations in $R^{n}$, Math. Zeit., Vol. 192, 1986, pp. 135-148.

[9] T. Kato, Strong $L^{p}$ Solutions of the Navier-Stokes Equations in $\mathbf{R}^{m}$ with Applications to Weak Solutions, Math. Zeit., Vol. 187, 1984, pp. 471-480.

[10] T. Kato and H. Fujta, On the non-stationnary Navier-Stokes system, Rend. Sem. Math. Univ. Padova, Vol. 32, 1962, pp. 243-260.

[11] T. Kato and H. Fusita, On the Navier-Stokes Initial Value Problem I, Arch. Rat. Mech. Anal., Vol. 16, 1964, pp. 269-315.

[12] J. Peetre, New thoughts on Besov Spaces, Duke Univ. Math. Series, Duke University, Durham, 1976.

[13] J. SERrIN, On the Interior Regularity of Weak Solutions of the Navier-Stokes Equations, Arch. Rat. Mech. Anal., Vol. 9, 1962, pp. 187-195.

[14] E. M. SteIn, Singular Integral and Differentiability Properties of Functions, Princeton University Press, 1970.

[15] M. TAYlor, Analysis on Morrey Spaces and Applications to Navier-Stokes and Other Evolution Equations, Comm. in PDE, Vol. 17, 1992, pp. 1407-1456.

[16] H. TRIEBEL, Theory of Function Spaces, volume 78 of Monographs in Mathematics, Birkhauser, 1983.

(Manuscript received October 24, 1994.) 